\title{
DISPOSICIÓN DE BASURAS PRODUCIDAS EN LAS AULAS DE CLASE POR PARTE DE LOS ESTUDIANTES DE LA UNIVERSIDAD SANTO TOMÁS
}

\author{
${ }^{1}$ Alex Yesith Cotes Jurado, ${ }^{2}$ Sonia Constanza Concha Sánchez \\ ${ }^{1}$ Estudiante $\mathrm{X}$ semestre $\mathrm{F}$. de Odontología U. Santo Tomás, Bucaramanga (Colombia). \\ ${ }^{2}$ Odontóloga U. Santo Tomás, Especialista en Educación y Comunicación para la Salud, MSc Epidemiología U. Industrial de Santander, \\ Candidata a Doctor U. Nacional de Colombia, Docente U. Santo Tomás, Bucaramanga (Colombia).
}

Autor responsable de la correspondencia: Sonia Constanza Concha Sánchez

Correo electrónico: sococosa@yahoo.com

\section{RESUMEN}

Objetivo: describir la disposición de basuras producidas en forma cotidiana en las aulas clase por parte de los estudiantes que desarrollan sus actividades académicas en el Campus de Floridablanca de la Universidad Santo Tomás.

Materiales y métodos: se realizó un estudio descriptivo de corte transversal en las aulas de clase de la Universidad Santo Tomás, sede Floridablanca. Se evaluaron variables como aula, horas de clases, edificio, día, jornada, semestre, facultad, nivel académico, asignatura, número de integrantes, grupo, botellas, envolturas de alimentos, vasos, papel, restos de alimentos, cantidad de depósitos en el aula y en el pasillo, y distancia de los depósitos. Se hizo un plan de análisis univariado y bivariado para realizar el cruce de variables, se usaron pruebas estadísticas como Chi cuadrado, test exacto de Fisher para las variables cualitativas, y prueba t de Student para las cuantitativas.

Resultados: se evaluaron 139 salones o aulas de clase, el 57,6\% (80) reportaban presencia de basura antes de iniciar clases y el 82\% (114) la tenían después de terminar la sesión educativa. La facultad que registró mayor aumento de presencia de basura después de terminar clases fue Cultura Física con el 69,2\% (9).

Conclusiones: los estudiantes de todos los semestres y de todas las facultades de la Universidad Santo Tomás tenían un manejo inadecuado de los desechos sólidos en las aulas de clase y utilizaban poco los depósitos de basura que estaban disponibles en cada una de las aulas. [Cotes AY, Concha SC. Disposición de basuras producidas en las aulas de clase por parte de los estudiantes de la universidad Santo Tomás. Ustasalud 2012; 11:11 - 18]

Palabras clave: Residuos sólidos, Educación ambiental, Manejo de basuras, Calidad de vida.

\section{WASTE DISPOSAL. PRODUCED IN CLASSROOMS BY STUDENTS AT SANTO TOMAS UNIVERSITY}

\section{ABSTRACT}

Objective: to describe how students in academic activities that develop at the Santo Tomas University manage the garbage in the classrooms on a daily basis.

Methods: a descriptive cross sectional study was done in the classrooms at Santo Tomas University in Floridablanca. Classrooms, school hours, name of the building, day, week, semester, faculty, academic level, subject, number of class members, group, bottles, food wrappers, cups, paper, food waste, amount of garbage dumps in classrooms and in hallways, and distance to these deposits were evaluated. An univariate and bivariate analysis using statistical tests such as Chi square, Fisher exact test for qualitative variables and Student's $t$ test for quantitative variables were done.

Results: one hundred thirty nine classrooms were evaluated $57.6 \%(80)$ reported the presence of garbage before starting classes and $82 \%$ (114) did after the academic session. The faculty that registered the highest increase of garbage after the classes end was physical culture with $69.2 \%$ (9).

Conclusions: students from all semesters and all the faculties at the Santo Tomas University showed an improper handling of solid waste in the classroom and seemed to use very little the garbage dumps that were available in are in each room.

Key words: Solid waste, Environment education, Waste management, Quality of life. 


\section{INTRODUCCIÓN}

Los residuos sólidos son producidos por las actividades diarias de los seres humanos; estos residuos aumentan cada día debido al crecimiento de la población, al consumismo y a la producción. Muchas personas generan basura sin tener conciencia del impacto que sus acciones tienen sobre el ambiente, algunas desconocen sobre la forma cómo desechar la basura por lo que varios medios de comunicación y educación han resaltado la importancia de ser responsables de esta problemática para manejarla apropiadamente.

El crecimiento poblacional influye directamente con el incremento en la producción de basura lo que representa un problema de tipo ecológico por la contaminación derivada del manejo inadecuado de residuos sólidos, económico porque la recolección y transporte de la basura representa costos cada vez más elevados y finalmente social por la necesidad de crear conciencia del problema de la basura y de la magnitud de las repercusiones. ${ }^{1,2}$ Se sabe que la disposición inadecuada de los desechos sólidos se debe a la falta de educación ambiental o cultura ambiental en las personas que al consumir producen la basura y la desechan en forma inadecuada. ${ }^{3}$

La educación ambiental se orienta al fortalecimiento de una cultura ambiental que involucre la creación de valores hacia el desarrollo sostenible y el cambio de actitud hacia el medio ambiente dentro de un plan de desarrollo que busca el mejoramiento de ambiente y la calidad de vida, por tanto, se requiere que las instituciones educativas adquieran una responsabilidad e incluyan en sus programas académicos la promoción de la preservación de un medio ambiente sano y el fomento de la educación y la cultura ecológica. ${ }^{4}$

Ull sugiere tres operaciones universitarias y tres ámbitos donde se introduce la ambientalización curricular, la investigación sobre el ambiente y la vida universitaria relacionada con ambientalizacion de las actividades diarias de la institución por lo que se debe tener en cuenta la comunicación, sensibilización con un voluntariado ambiental y cooperación. ${ }^{5}$ Desde los años 90 las universidades empezaron a plantear la aplicación de criterios ambientales de forma global que incluyen aspectos como la energía, agua, medio natural, materiales, residuos, calidad de aire interior de los edificios iluminación natural y todas las fases de edificación, diseño, construcción y utilización. ${ }^{5}$

En Colombia, la preocupación por los residuos sólidos generados en las zonas urbanas ha partido de consideraciones de tipo higiénico y sanitario. ${ }^{6} \mathrm{El}$ problema comenzó cuando la comunidad empezó a dejar los residuos sólidos en las vías públicas y esperaba que alguien los retirara. Lo que llevó al análisis sectorial liderado por el Ministerio del Medio Ambiente con el apoyo de la Organización Panamericana de la Salud (OPS), la Organización Mundial de la Salud (OMS) y el Banco Mundial, Ministerio de Desarrollo Económico y de Salud, del Departamento Nacional de Planeación a través de su Unidad de Política Ambiental y otros para profundizar la gestión integral de residuos sólidos en Colombia que incluían los peligrosos y los no peligrosos. De esta manera, se identificó que la problemática ambiental de los residuos sólidos se asocia a seis aspectos fundamentales: práctica inadecuada en la disposición final de los residuos; el manejo de los residuos sólidos depende sólo de la prestación del servicio domiciliario del aseo; generación creciente de residuos y deficiencias en el aprovechamiento y valoración de los mismos; bajo desarrollo industrial del sector; falta de educación y participación ciudadana en el manejo ambiental de los residuos y ausencia de conocimientos sobre la magnitud del problema.

Esta situación muestra la urgente necesidad de ayudar, desde las universidades, en la implementación y ejecución de los programas de gestión de residuos sólidos a través de la investigación con el objetivo de impedir o minimizar los riesgos que para los seres humanos y el ambiente ocasionan los residuos sólidos y peligrosos. Así se contribuirá a la protección ambiental eficaz y al crecimiento económico de nuestro país.

\section{MATERIALES Y MÉTODOS}

Se realizó un estudio observacional descriptivo de corte transversal. ${ }^{7}$ El universo lo conformaron las 845 aulas de clase o salones ubicados en los edificios Santander y Fray Angélico de la Universidad Santo Tomás, Campus Floridablanca (Santander).

La obtención de la muestra se calculó mediante la rutina simple size del paquete Epi Info 6.04. De esta manera se obtuvieron 139 salones de los dos edificios mencioandos. ${ }^{8}$ Las siguientes especificaciones de diseño para un estudio descriptivo de corte transversal fueron consideradas: el total de salones del edifico Santander y Fray Angélico de la sede Floridablanca (845), la prevalencia esperada de los salones con disposición de desechos inadecuados del $50 \%$, un peor resultado esperado de $40 \%$ y un nivel de confianza de $99 \%$ lo que indicó que 139 salones debían ser evaluados. La selección se hizo por muestreo probabilístico de tipo aleatorio simple.

Fueron incluidos en el estudio aulas o salones de clase en los que se imparten clases de pregrado y posgrado ubicados en los edificios Santander y Fray Angélico de la Universidad Santo Tomás en el cam- 
pus Floridablanca, torreones y aulas especiales en los que se desarrolla la actividad académica para los estudiantes de la Universidad. Como criterios de exclusión se definieron los salones del área de dibujo, auditorios, aulas de informática, preclínicas y clínicas de odontología y optometría del edificio Santander y Fray Angélico.

Las variables se registraron en un instrumento diseñado para este fin. La variable de salida fue presencia de basura antes de clases y presencia de basura después de clases que se recolectó mediante la observación de cada salón evaluado. Como variables socio-demográficas se consideraron el salón de clase, la hora de entrada y la de salida, el edificio, el día de la semana, el total de horas de clase, la jornada, el semestre, la facultad, el nivel académico, la asignatura, el número de integrantes del grupo, el grupo, la cantidad de depósitos de basura en el salón, la cantidad de depósitos de basura en el pasillo y distancia a la que se encontraban los depósitos de basura. Entre la variable de presencia de basura se tuvo en cuenta los tipos de residuos sólidos que se encontraban en el salón de clase: botellas, envolturas de alimentos, vasos o pocillos desechables, papelería y restos de alimentos.

Los procedimientos incluyeron la realización de una prueba piloto para entrenar al evaluador y estandarizar los criterios de observación para cada una de las variables contempladas en el formulario. Se verificó la eficacia del instrumento, se estimaron los tiempos y se revisaron las variables contempladas. Se realizó una previa recolección de la información y se inició con las variables socio-demográficas y, posteriormente, se evaluó la variable presencia de basura antes y después de la clase.

Una vez recolectada la información se procedió a efectuar la codificación de cada uno de los instrumentos y la sistematización por duplicado, en el programa Excel la información obtenida se exportó al programa EPI-INFO 6.04 y mediante la rutina $v a-$ lídate se procedió a verificar la calidad de la digitación de la misma. ${ }^{8,9}$ Se efectuaron las correcciones necesarias y la base depurada se exportó al paquete estadístico STATA 9.1 para su correspondiente procesamiento. ${ }^{10}$ Se hizo un plan de análisis univariado y bivariado para realizar el cruce de variables al utilizar las pruebas estadísticas Chi cuadrado y test exacto de Fisher para las variables cualitativas, y la prueba t de Student para las cuantitativas. ${ }^{11}$ Todo el análisis se realizó con un nivel de significancia alfa $(\alpha)=0.05$.

De acuerdo con la Resolución 008430 de 1993, emitida por el Ministerio de Salud en la cual se exponen las normas científicas, técnicas y administrati- vas que debe tener un proyecto de investigación al trabajar en comunidades y en los artículos 17-22, se protege constantemente la privacidad, no existe riesgo alguno previsto, existe completo conocimiento y responsabilidad por parte de los investigadores de todas las acciones llevadas a cabo y si se llegase a ocasionar daño la institución o los mismos investigadores están dispuestos a ofrecer atención inmediata. ${ }^{12}$

\section{RESULTADOS}

Se evaluaron 139 salones vinculados con 112 asignaturas de seis facultades, todas estas de pregrado que cursan sus asignaturas académicas en la Universidad Santo Tomás, en el Campus Floridablanca. El 57,6\% (80) reportaban presencia de basura antes de iniciar clases y el 82\% (114) la tenían después de terminar la sesión educativa (Tabla 1).

El 62,1\% (36) de los salones del edificio Fray Angélico presentó basura antes de comenzar clases y aumentó a 96,5\% (56) después de terminar las clases. Al revisar el día de la semana que tuvo mayor presencia de basura antes de comenzar clases fue el día miércoles con el 78,3\% (18) que aumentó al 95\% (22) después de terminar clases. Cabe resaltar, que dentro de los días de la semana, el que menos tuvo presencia de basura antes de clase fue el día viernes con un 35\% (12) que aumentó a un 58\% (20) después de terminar clase. Es necesario aclarar que en este día se evaluaron más salones que el resto de los días de la semana. Se evaluó el 57,6\% (80) en la jornada de la mañana, con $68,8 \%$ (55) de presencia de basura antes de comenzar clases y aumentó a $87,5 \%$ (70) después de terminar clases. (Tabla 1)

El semestre que más reportó presencia de basura antes de comenzar clase fue el primer semestre con un $79,2 \%$ (19) que aumentó al $91,7 \%$ (22) después de terminar. En general, séptimo semestre registró $75 \%$ (6) de presencia de basura antes de iniciar clase el cual aumentó al 100\% (8) después de terminar clase. Es importante anotar que décimo semestre tuvo un $50 \%$ (2) de presencia de basura antes de comenzar clase y aumentó a 75\% (3) después de terminar las clases lo cual muestra que el problema del manejo inadecuado de los desechos se presenta desde el primer semestre hasta décimo semestre (Tabla 1).

La facultad que registró mayor aumento de presencia de basura después de terminar clases fue Cultura Física con $69.2 \%$ (9). Se aclara que la facultad de Odontología y Optometría a pesar de ser facultades de ciencias de la salud presentan un aumento de basura después de terminar clases, Odontología con el $82,7 \%$ (43) y Optometría con el $100 \%$ (17) (Tabla 1). 
Dentro de los desechos sólidos evaluados se encontró que las botellas, envolturas y papel registraron asociaciones estadísticamente significativas al comparar el antes y el después de clase. El promedio de botellas encontradas en los salones antes de comenzar clases fue de 0,06 $\pm 0,24$ que aumentó después de clases a $0,18 \pm 0,44(p=0,0001)$, el de las envolturas fue de 1,4 $\pm 2,1$ que aumentó después de clases a 2,0 $\pm 2,2(p=0,0001)$ y el promedio de papel encontrado en los salones antes de comenzar clases fue de 2,7 $\pm 3,7$ que aumentó después de clases a $4,1 \pm 5,4(p=0,0004)$ (Tabla 2).

Para el análisis de los factores que pueden influir en el manejo inadecuado de los desechos sólidos en los salones de la Universidad Santo Tomás en Floridablanca no se observó ninguna asociación con la intensidad horaria, integrantes de grupo, depósitos de basura en el salón y pasillo, ni la distancia a la que estos se encontraban (Tabla 3).

Tabla 1. Descripción de aspectos relacionados con salones evaluados, días, jornadas, semestre y facultades. Usuarios de los salones.

\begin{tabular}{|c|c|c|c|c|c|c|c|}
\hline \multirow[t]{2}{*}{ Variables } & \multirow{2}{*}{$\begin{array}{c}\text { Global } \\
\text { n (\%) }\end{array}$} & \multicolumn{2}{|c|}{$\begin{array}{l}\text { Presencia de basura } \\
\text { antes de clases }\end{array}$} & \multirow[t]{2}{*}{$\mathbf{P}^{*}$} & \multicolumn{2}{|c|}{$\begin{array}{l}\text { Presencia de basura } \\
\text { después de clases }\end{array}$} & \multirow[t]{2}{*}{$\mathbf{P}^{*}$} \\
\hline & & $\mathrm{Si}$ & No & & Si & No & \\
\hline Población & $139(100)$ & $80(57,6)$ & $59(42,4)$ & & $114(82)$ & $25(18)$ & \\
\hline \multicolumn{8}{|l|}{ Edificio } \\
\hline Santander & $81(58,3)$ & $44(54,3)$ & $37(45,7)$ & 0,362 & $58(71,6)$ & $23(28,4)$ & $0,0001+$ \\
\hline Fray Angélico & $58(41,7)$ & $36(62,1)$ & $22(37,9)$ & & $56(96,5)$ & $2(3,45)$ & \\
\hline \multicolumn{8}{|c|}{ Día de la semana } \\
\hline Lunes & $26(18,7)$ & $14(53,9)$ & $12(46,2)$ & $0,010+$ & $20(76,9)$ & $6(23,1)$ & $0,001+$ \\
\hline Martes & $31(22,3)$ & 18 (581) & $13(41,9)$ & & $29(93,5)$ & $2(6,5)$ & \\
\hline Miércoles & $23(16,6)$ & $18(78,3)$ & $5(21,7)$ & & $22(95,6)$ & $1(4,4)$ & \\
\hline Jueves & $25(18,0)$ & $18(72,0)$ & $7(28,0)$ & & $23(92,0)$ & $2(8,0)$ & \\
\hline Viernes & $34(24,5)$ & $12(35,3)$ & $22(64,7)$ & & $20(58,8)$ & $14(41,2)$ & \\
\hline \multicolumn{8}{|l|}{ Jornada } \\
\hline Mañana & $80(57,6)$ & $55(68,8)$ & $25(31,3)$ & $0,002+$ & $70(87,5)$ & $10(12,5)$ & $0,050+$ \\
\hline Tarde & $59(42,5)$ & $25(42,4)$ & $34(57,6)$ & & $44(74,6)$ & $15(25,4)$ & \\
\hline \multicolumn{8}{|l|}{ Semestre } \\
\hline Primero & $24(17,3)$ & $19(79,2)$ & $5(20,8)$ & 0,131 & $22(91,7)$ & $2(8,3)$ & 0,456 \\
\hline Segundo & $18(12,9)$ & $10(55,6)$ & $8(44,4)$ & & $14(77,8)$ & $4(22,2)$ & \\
\hline Tercero & $15(10,8)$ & $5(33,3)$ & $10(66,7)$ & & $10(66,7)$ & $5(33,3)$ & \\
\hline Cuarto & $15(10,8)$ & $7(46,7)$ & $8(53,3)$ & & $12(80,0)$ & $5(20,0)$ & \\
\hline Quinto & $16(11,5)$ & $10(62,5)$ & $6(37,5)$ & & $14(87,5)$ & $2(12,5)$ & \\
\hline Sexto & $16(11,5)$ & $10(62,5)$ & $6(37,5)$ & & $14(87,5)$ & $2(12,5)$ & \\
\hline Séptimo & $8(5,8)$ & $6(75,0)$ & $2(25,0)$ & & $8(100,0)$ & --- & \\
\hline Octavo & $16(11,5)$ & $6(37,5)$ & $10(62,5)$ & & $11(68,8)$ & $5(31,3)$ & \\
\hline Noveno & $7(5,0)$ & $5(71,4)$ & $2(28,6)$ & & $6(85,7)$ & $1(14,3)$ & \\
\hline Décimo & $4(2,9)$ & $2(50,0)$ & $2(50,0)$ & & $3(75,0)$ & $1(25,0)$ & \\
\hline \multicolumn{8}{|l|}{ Facultad } \\
\hline Odontología & $52(37,4)$ & 31 (59.6) & $21(40,4)$ & 0,165 & $43(82,7)$ & $9(17,3)$ & 0,245 \\
\hline Optometría & $17(12,2)$ & $13(76.5)$ & $4(23,5)$ & & $17(100,0)$ & --- & \\
\hline Derecho & $46(33,1)$ & $25(54.4)$ & $21(45,7)$ & & $36(78,3)$ & $10(21,7)$ & \\
\hline L. Dental & $2(1,4)$ & $2(100)$ & --- & & $2(100,0)$ & --- & \\
\hline Cultura Física & $13(9,3)$ & $4(30.8)$ & $9(69,2)$ & & $9(69,2)$ & $4(30,8)$ & \\
\hline Arquitectura & $5(3,6)$ & $2(40,0)$ & $3(60,0)$ & & $4(80,0)$ & $1(20,0)$ & \\
\hline Q. Ambiental & $4(2,9)$ & $3(75,0)$ & $1(25,0)$ & & $3(75,0)$ & $1(25,0)$ & \\
\hline \multicolumn{8}{|l|}{ Nivel académico } \\
\hline Pregrado & $139(100)$ & $80(57.6)$ & $59(42.5)$ & & $114(82)$ & 25 (17.99) & \\
\hline
\end{tabular}

$\alpha=0.05{ }^{*}$ Chi cuadrado **Test exacto de Fisher +Estadísticamente significativo 
Tabla 2. Descripción de presencia de basura antes y después de clase.

\begin{tabular}{|c|c|c|c|}
\hline \multirow{2}{*}{ Variables } & \multicolumn{2}{|c|}{ Clases frecuencia $(\mathrm{x})$} & \multirow{2}{*}{$\mathbf{P}^{*}$} \\
\hline & Antes $(\mathrm{x}) \pm$ D.E & Después $(\mathrm{x}) \pm$ D.E & \\
\hline Botellas & $0,06 \pm 0,24$ & $0,18 \pm 0,44$ & $0,0001+$ \\
\hline Envolturas & $1,40 \pm 2,10$ & $2,00 \pm 2,20$ & $0,0001+$ \\
\hline Papel & $2,70 \pm 3,70$ & $4,10 \pm 5,40$ & $0,0004+$ \\
\hline Restos de alimentos & $0.09 \pm 0.85$ & $0,02 \pm 0,16$ & 0,3724 \\
\hline Vasos desechables & $0,08 \pm 0,32$ & $0,10 \pm 0,04$ & 0,2867 \\
\hline
\end{tabular}

Tabla 3. Descripción de presencia de basura según la intensidad horaria, integrantes de grupo, número de depósitos en el pasillo y distancia de depósitos de basura en el salón de clases.

\begin{tabular}{|c|c|c|c|}
\hline \multirow{2}{*}{ Variables } & \multicolumn{2}{|c|}{ Presencia de Basura frecuencia (x) } & \multirow{2}{*}{$\mathbf{P}^{*}$} \\
\hline & $\mathrm{Si}$ & No & \\
\hline Total horas de clases & $103,6 \pm 40,4$ & $109,3 \pm 36,8$ & 0,395 \\
\hline Integrantes del grupo & $21,6 \pm 6,3$ & $22,6 \pm 5,6$ & 0,371 \\
\hline Número depósitos basura pasillo & $1,9 \pm 0,9$ & $2,1 \pm 0,9$ & 0,292 \\
\hline $\begin{array}{l}\text { Distancia depósitos de basura salones de } \\
\text { clases }\end{array}$ & $2,7 \pm 0,7$ & $2,8 \pm 0,6$ & 0,398 \\
\hline
\end{tabular}

\section{DISCUSIÓN}

En el presente estudio se observó que la disposición inadecuada de las basuras en los salones o aulas de clase por parte de los estudiantes, es una situación que afecta a la comunidad universitaria del Campus Floridablanca de la Universidad Santo Tomás.

Jacobson en un proceso desarrollado en la Universidad Estatal de la Florida hace énfasis en cómo las universidades generan gran cantidad de residuos de todo tipo y cuya disposición representa grandes costos para las instituciones ${ }^{13}$ además, menciona la obligación moral de llevar a cabo operaciones responsables que deben ser asumidas por todas y cada una de las personas que integran la comunidad universitaria.

En la literatura revisada, no se evidenciaron muchos estudios que permitieran comparar los resultados obtenidos con el presente trabajo; sin embargo, es necesario resaltar la importancia de desarrollar este tipo de estudios, pues de acuerdo a lo expresado por Olarte y citado por Brenen, ${ }^{14}$ la educación debe visualizarse como un sistema abierto que no se puede aislar del mundo sociocultural y será el resultado de un proceso de interacción con él.

La disposición de desechos sólidos se constituye en una responsabilidad ineludible de cada ser humano que vive en este planeta y del que no pueden desligarse los sistemas de educación superior y avanzada, pues el daño sobre el ecosistema tiene profundas consecuencias en el presente y serias re- percusiones para el futuro de cada uno de nosotros y de las generaciones que vendrán.

En este estudio se evidencia una gran problemática relacionada con el manejo inadecuado de los desechos sólidos puesto que los estudiantes de primero hasta décimo semestre vinculados a las seis facultades evaluadas, hicieron un manejo inadecuado de éstos, se observó que en el 57,6\% de los salones se evidenciaban basuras al iniciar las clases y al finalizar éstas en el $82 \%$, por lo que se sugiere que los estudiantes depositan una buena cantidad de residuos durante el desarrollo de las asignaturas.

En un reporte de la Universidad Estatal de Míchigan se observó cómo en las aulas de clase, los salones de lectura y los laboratorios de computadores, usuales espacios de trabajo de sus estudiantes, se recolecta una buena cantidad de restos de papel, periódicos, botellas plásticas y latas. Sin embargo, en ese documento no establecen la cantidad de residuos, aunque, el tipo de basura encontrada si es muy similar al observado en el presente trabajo. ${ }^{15}$ En esta investigación, el residuo sólido más frecuentemente observado fue restos de papel encontrándose un promedio de tres al iniciar la clase que aumentó a cuatro al terminar la asignatura; se destacan además las envolturas, usualmente de alimentos, encontrándose en promedio una envoltura al inicio de la clase que aumentó a dos al terminar ésta; también se encontraron botellas, restos de alimentos y vasos desechables. 
Esto podría ser el reflejo de una falta de cultura institucional relacionada con la educación en el manejo de basuras, no sólo por parte de los estudiantes sino también de los profesores que no inculcan a los estudiantes el buen manejo de los desechos. Adicionalmente, no se puede desconocer la influencia que el individualismo y el egoísmo puedan generar frente a este fenómeno. Berbenaste y colaboradores en su trabajo Grado de percepción de anomia en estudiantes universitarios, reportaron cómo el comportamiento de los jóvenes está orientado fundamentalmente a satisfacer los intereses personales, la indiferencia ante la autoridad y el otro, y el apego a las cosas superfluas que se constituyen en los aspectos más importantes y que determinan sus acciones cotidianas. ${ }^{16}$

Esto repercute en los diferentes ámbitos en los que transcurre la vida, dentro de los que cabría destacar el manejo privado que se hace del espacio público. Así también resaltaron cómo estos espacios son convertidos (por las personas que transitan por ellos) en depósitos de basura lo que consideraron anormal e incluso correcto.

La indiferencia de los jóvenes universitarios podría reflejarse en el hecho que, cada salón cuenta con un depósito de basura. Sin embargo, a los estudiantes no les preocupa dejar la basura en el suelo, sobre las mesas o las sillas de trabajo que existen en cada aula de clase, sin importarles que las personas de su comunidad, que posteriormente usaran el salón, lo encuentren en condiciones inadecuadas.

Es importante considerar, que la presencia de basuras en los espacios públicos que se comparten con otros miembros de la comunidad universitaria son quizás el reflejo de la creencia que éstos pueden ser dejados de cualquier manera, con la convicción que siempre existirá alguien responsable de la limpieza de ellos, sin pensar que la tarea de mantener el aseo en los salones y todos los espacios que se utilizan es un trabajo que parte de asumir en forma personal una actitud responsable frente al manejo adecuado de los desechos sólidos que se producen en la vida cotidiana.

En el trabajo de Vásquez y colaboradores desarrollado en la Facultad de Medicina de la Universidad Autónoma de Nicaragua y en la que se analizó la percepción que tienen los docentes, administradores y estudiantes sobre la acumulación de basuras y la suciedad en su facultad de salud, se encontró que tanto los docentes como los administradores consideraron que las condiciones inadecuadas se derivaban, de la "haraganería" de los estudiantes para usar los depósitos de basura. Los estudiantes por su parte, responsabilizan de esta situación a la "negligencia" de las personas responsables de recolectar los desechos. ${ }^{17}$ Aspectos que deberían ser explorados en próximas investigaciones, relacionadas con el tema, que se desarrollen en la Universidad Santo Tomás pues para implementar un programa orientado a fortalecer una cultura en el manejo de basuras, se debe partir de identificar no sólo las prácticas sino también las creencias que sobre el tema se tienen.

$\mathrm{Al}$ analizar la posibilidad de generar una cultura institucional en el manejo de basuras en el ámbito universitario es importante retomar a Brenes y colaboradores, quienes resaltaron cómo la educación ambiental le atañe no sólo a los estudiantes universitarios; ${ }^{14}$ es importante que las instituciones de educación superior, sus directivas, los responsables del sector administrativo y sus profesores comprendan que no sólo se le debe enseñar a los estudiantes el conocimiento técnico profesional necesario para desempeñarse fuera de esta, sino que deben brindarle habilidades y marcos de referencia que les ayuden a actuar como personas responsables, capaces de adaptarse al medio en que se encuentren y generar espacios que les permitan cambiar aquellos que puedan ser nocivos para sí o para las personas con quienes conviven.

Como tal, todos los estudiantes deben tener un espacio de formación para desarrollar una conciencia ambiental, un pensamiento crítico e interés y capacidad para actuar frente a situaciones inadecuadas que afecten su vida y la de sus comunidades; con la capacidad y la responsabilidad de generar alternativas, para que todas las personas puedan mejorar su calidad de vida con una postura ética, responsable y solidaria. También, es necesario dejar de lado ese pensamiento de que todo lo relacionado con el ambiente sólo es para los ambientalistas y debe convertirse en parte del lenguaje cotidiano y de la formación universitaria.

Al considerar lo expresado por García quien retoma a Coley, se evidenció que los conocimientos ambientales se adquieren a lo largo de la vida de los seres humanos; fundamentos que se acumulan a partir de diferentes fuentes como la escuela, las lecturas personales, los amigos, las bases y valores que provee la familia, las experiencias personales y, por supuesto, de los medios masivos de comunicación; claro está, que los conceptos que se desarrollan a partir de estas fuentes pueden dar como resultado creencias y pensamientos erróneos, así como actitudes y conductas ambientales poco favorables. ${ }^{18}$ Es necesario que en las universidades se solidifiquen los conocimientos confusos que se presentan en los estudiantes sobre el manejo de los desechos sólidos 
en las aulas de clases y se incentive a los estudiantes para así llegar a una formación integral tanto en lo profesional como en lo social.

González evaluó el conocimiento sobre el ambiente y el medio ambiente en estudiantes de primer y segundo año de la carrera de Ecología y de segundo año de Licenciatura en Ciencias del Ambiente (ambas en universidades de Buenos Aires, Argentina), y encontró que a pesar del nivel educativo únicamente el 1\% de los estudiantes tenía una definición compleja sobre el medio ambiente, entre el 14\% y $44 \%$ tenía una visión indiferenciada, mientras que el $33 \%$ y el $52 \%$ identifica componentes, pero no establece relaciones entre ellos. ${ }^{19} \mathrm{Al}$ analizar los resultados de este estudio, se podría sugerir que algo pasa en relación con los conocimiento de los estudiantes y algo falla en el proceso educativo que se lleva a cabo en los grupos evaluados. Es importante considerar que la misma situación observada por González puede estar ocurriendo en la Universidad Santo Tomás pero sería necesario un estudio donde se analice y se observe tanto el conocimiento de los estudiantes como el comportamiento hacia el medio ambiente, porque al conocer bien estas bases fácilmente se podría tener un manejo adecuado de los desechos sólidos.

Los hallazgos de este trabajo plantean la necesidad de evaluar los salones, el comportamiento e incluso el conocimiento sobre el manejo adecuado de los desechos por parte de los estudiantes de la Universidad. Es necesario crear conciencia en los mismos para que conozcan la importancia que tiene el manejo adecuado de los desechos sólidos en el hogar, en el lugar de trabajo y en todos los espacios de la vida.

Es importante mencionar que se observó que la facultad que presentó mayor aumento de basura después de terminar clase, fue Cultura Física pues pasó de $31 \%$ a un $70 \%$. Las facultades de salud como Odontología y Optometría también registraron un aumento, pues en la primera se pasó de $60 \%$ a $83 \%$ y en la segunda, de $77 \%$ a $100 \%$. Es importante anotar que en las facultades relacionadas con salud esto es una problemática seria pues dentro de sus asignaturas se deberían desarrollar actividades orientadas a fortalecer las normas de bioseguridad y disposición adecuada de desechos como parte de una práctica profesional idónea.

En relación con las facultades de Ciencias de la Salud se debe tener presente lo expresado por Baldi y García quienes retoman el documento publicado por la OMS (1994) en el que se expresó que dentro del proceso de formación de los profesionales de la salud se debía proveer una base en la que prime la ideología del bienestar común y el interés por el otro. En el proceso de construcción de la salud, la posición de las personas en la vida no debe percibirse desde el aspecto individual, sino que tiene que abordarse económica, social, cultural y ambientalmente de manera integral al ser responsabilidad de sus profesionales desarrollar prácticas saludables consecuentes con el proceso de formación que desarrollan con sus pacientes o las personas a las que abordan. ${ }^{20}$

Sin embargo, esto no exime a los estudiantes de las áreas de las humanidades o las tecnológicas, entre otras, de asumir una conducta responsable frente a su salud y su ambiente. No se entiende el por qué se presentó un aumento en la presencia de basura en el transcurso de las clases en cualquiera de las facultades evaluadas, más aún si se retoma la Misión de la Universidad Santo Tomás ya en ella se evidencia el interés de la Institución por promover la formación integral de las personas, en el campo de la Educación Superior, mediante acciones y procesos de construcción de conocimiento, investigación y proyección social, para que respondan de manera ética, creativa y crítica a las exigencias de la vida humana y estén en condiciones de aportar soluciones a la problemática y necesidades de la sociedad y del país. ${ }^{21}$ Con esto en mente, se considera que debe haber un compromiso institucional y de la comunidad académica en general para responder a la problemática en la disposición inadecuada de los desechos sólidos en forma pertinente y oportuna.

Cabe considerar como limitantes de este estudio no haber explorado los conocimientos que tenían los estudiantes sobre la educación ambiental en las diferentes facultades de la Universidad. Adicionalmente, no haber incluido en este estudio, la evaluación de las prácticas relacionadas con la disposición de basuras en salones de clase de los estudiantes de los diferentes posgrados que se cursan en esta sede y que de acuerdo a observaciones informales, podrían tener un comportamiento aún peor en cuanto al manejo de basuras durante el desarrollo de sus clases presenciales, al observado en los grupos de pregrado.

En la Universidad Pedagógica Nacional la manera de ofrecer una educación ambiental es a través de un curso estándar único dictado para todos los estudiantes por medio de medios masivos de enseñanza como la televisión, videos multicopiados y materiales impresos de auto-estudio, para posteriormente hacer discusiones con los docentes de las diferentes facultades. El punto de partida de esta Universidad era vincular la dimensión ambiental en todas las carreras académicas a través de un núcleo básico de 
formación de los docentes. ${ }^{22}$ Es posible involucrar la formación del docente en investigaciones que aborden los distintos problemas relacionados con la ciencia, la tecnología y la pedagogía desde el pensamiento ambientalista para promover el desarrollo de trabajos de grado y de proyectos de investigación de pregrado en el área ambiental dirigidos por comités interdisciplinarios.

Cabría preguntarse entonces sí: ¿A mayor nivel educativo peor es nuestro comportamiento en cuanto a la disposición de desechos sólidos en los salones de clase? ¿Es posible que pasemos por la Universidad sin que ésta pase por nosotros? ¿Es responsabilidad de las Universidades promover la educación ambiental en sus estudiantes tanto de pregrado como de posgrado independiente de su área de formación?

Se podría concluir que los estudiantes de todos los semestres y de todas las facultades de la Universidad Santo Tomás tenían un manejo inadecuado de los desechos sólidos en las aulas de clase y parecía que utilizaban poco los depósitos de basura dispuestos en cada salón. Es necesario promover el uso de los depósitos de basura en los salones y pasillos de la Universidad por parte de los directivos, administrativos, profesores, estudiantes, personal de mantenimiento y servicios generales con el fin de estimular una responsabilidad comunitaria y conciencia social, ambiental y cultural.

Este estudio mostró la necesidad de realizar una investigación para anlizar el conocimiento ambientalista de los estudiantes y así corroborar si en realidad el manejo inadecuado de los desechos sólidos es consecuencia de la falta de educación ambiental en ellos. Adicionalmente, sería importante diseñar programas orientados a promover el manejo adecuado de los desechos sólidos por medio de ilustraciones fotográficas, frases sobre la problemática ambiental que se viven en la sociedad, así como de las causas, consecuencias económicas, sociales y ambientales de este mal uso de los depósitos de desechos sólidos.

\section{BIBLIOGRAFÍA}

1. Ojeda S, Muñoz R, González FF. Análisis estadístico del comportamiento de los residuos sólidos domiciliarios en una comunidad urbana. Frontera Norte 1998; 10: 65 - 76.

2. Collazos H. Gestión integral de residuos sólidos la regionalización de los rellenos sanitarios una visión hacia la formulación de política pública 2007; 8 .

3. Programa Institucional Universidad Nacional (UNA), UNA-Campus Sostenible, 2007.

4. Paz V, Jiménez ML, Méndez MM. La educación ambiental en la Universidad Pedagógica Nacional. Red Académica, Horizontes Educativos; 2010.
5. Ull Á. El impacto de la actividad universitaria sobre el medio ambiente. Revista Eureka sobre Enseñanza y Divulgación de las Ciencias 2008; 5: 356 - 366.

6. Medina CI. Manejo de residuos sólidos. Revistas de la Facultad de Ingeniería 1999.

7. Morillo L. Epidemiologia clínica. Investigación clínica aplicada. Editorial Médica Panamericana, S.A. 2004.

8. CDC - OMS. Epi - Inf 6. Versión 6.04. Nov 1996.

9. Microsoft Excel 5.0. Microsoft Corporation 1998.

10. Stata Corp 2003. Stata Statistical Software release 8.0 college station, Texas.

11. Norman GR, Streiner Dl. Bioestadistica. Mosby/Doyma. Libros. Madrid 1996 p 129 - 147.

12. República de Colombia. Ministerio de Salud. Resolución No. 008430 de 1993. Título II de la investigación en Seres Humanos, Capítulo 1 de los Aspectos Éticos de la Investigación en Seres Humanos. Artículos 5 - 16.

13. Jacobson TL. Solid waste management at Florida State University: an analysis of option. Florida State University. Tallahassee, Florida December 2002

14. Brenes Miriam, Chaverri O, Gaviria L. Eje transversal ambiental en el Instituto Tecnológico de Costa Rica. Tecnología en Marcha 2008; 21: 3 - 8.

15. Selki SE, Joshi S. Sidique S, Dabholkar G. White paper on campus solid waste. Michigan State University. March 14, 2006

16. Benbenaste N, Etchezahar E, Costa G, Petit L. Grado de percepción de anomia en estudiantes universitarios. Revista Académica Hologramática 2008: 109-12. URL disponible en: http: www.hologramatica.com.ar

17. Vásquez H, Cardoza U, Cortés M. Diseño de intervención sobre salud ambiental de la Facultad de Medicina UES. Universidad Nacional Autónoma de Nicaragua. San Salvador. 1998

18. García M. Los conocimientos ambientales de estudiantes universitarios. URL disponible en: http://www.comie.org. $\mathrm{mx} /$ congreso/memoriaelectronica/v09/ ponencias/at03/ PRE1176230766.pdf

19. González E. Las concepciones del medioambiente en estudiantes de nivel superior. Revista Iberoamericana de Educación. URL disponible en: http://www.rieoei.org/ deloslectores/602Gonzalez.PDF

20. Baldi G, García E. Calidad de vida y medio ambiente, la psicología ambiental. Universidades 2005; 30: 9 - 16.

21. Universidad Santo Tomás. Misión de la Universidad Santo Tomás. URL disponible en: http: web.ustabuca.edu.co/ inicio/academia/documentosins/index.jsp.

\section{Correos electrónicos de los autores:}

Alex Yesith Cotes Jurado: alexyesith1@hotmail.com Sonia Constanza Concha Sánchez: sococosa@yahoo.com 